\title{
Incomplete compensation to articulatory perturbation
}

David H. McFarland, and Shari R. Baum

Citation: The Journal of the Acoustical Society of America 97, 1865 (1995); doi: 10.1121/1.412060

View online: https://doi.org/10.1121/1.412060

View Table of Contents: https://asa.scitation.org/toc/jas/97/3

Published by the Acoustical Society of America

\section{ARTICLES YOU MAY BE INTERESTED IN}

Speech compensation to structural modifications of the oral cavity

The Journal of the Acoustical Society of America 100, 1093 (1996); https://doi.org/10.1121/1.416286

Production of bite-block vowels: Acoustic equivalence by selective compensation

The Journal of the Acoustical Society of America 69, 802 (1981); https://doi.org/10.1121/1.385591

Learning to produce speech with an altered vocal tract: The role of auditory feedback

The Journal of the Acoustical Society of America 113, 532 (2003); https://doi.org/10.1121/1.1529670

Sensorimotor adaptation to feedback perturbations of vowel acoustics and its relation to perception The Journal of the Acoustical Society of America 122, 2306 (2007); https://doi.org/10.1121/1.2773966

Compensation to articulatory perturbation: Perceptual data

The Journal of the Acoustical Society of America 99, 3791 (1996); https://doi.org/10.1121/1.414996

The input/output formula: A theoretical approach to the fitting of personal amplification devices

The Journal of the Acoustical Society of America 97, 1854 (1995); https://doi.org/10.1121/1.412980

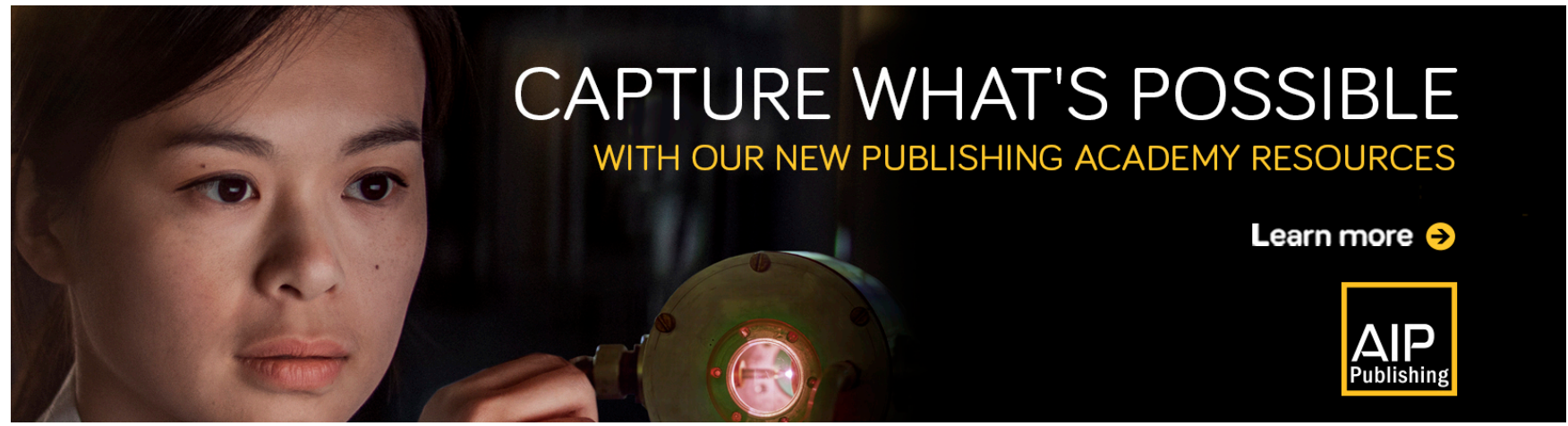




\title{
Incomplete compensation to articulatory perturbation
}

\author{
David H. McFarland \\ École d'orthophonie et d'audiologie, Faculté de médecine, Université de Montréal, Montréal, Canada \\ Shari R. Baum \\ School of Communication Sciences and Disorders, Faculty of Medicine, McGill University, Canada
}

(Received 18 January 1994; accepted for publication 15 November 1994)

\begin{abstract}
Measurements were made of vowels, fricatives, and stop consonants produced by 15 adult speakers of French in one free-mandible and two fixed mandible conditions. Speech acoustic data were recorded immediately upon bite-block insertion and after a 15-min accommodation period. Results indicate that compensation to increased jaw opening during speech is neither immediate nor complete as there were small but significant differences in the acoustic parameters of vowels and consonants produced under bite-block and normal conditions. Further, the data suggest that, at least for vowels, speech compensatory strategies may develop over time, perhaps involving error-based correction. Consonants appear to require a more lengthy period of speech adaptation, and this may be due to the articulatory requirements for their accurate production. Individual differences in compensatory abilities are also discussed.
\end{abstract}

PACS numbers: 43.70.Aj

\section{INTRODUCTION}

The literature on the motor control of speech is replete with references to the variability and context dependency of articulation. There are a number of articulatory configurations that can give rise to the same acoustic percept and, moreover, there are a number of ways to achieve a given articulatory configuration. In fact, most theories of speech motor control incorporate in some fashion the notion of "motor equivalence," which may be defined as variable muscle activation patterns giving rise to the same movement goal (Abbs, 1973; Abbs and Netsell, 1973; Hughes and Abbs, 1976; MacNeilage, 1970). Further, any theory of speech motor control must take into account the large body of evidence of compensatory articulation to a variety of perturbations to the oral environment (Abbs, 1986; Hamlet and Stone, 1976; Kelso and Tuller, 1983; Lindblom et al., 1979; Warren et al., 1984). In the absence of such compensation, alterations and/or deviations in the physical characteristics of the vocal tract would change the resonant characteristics of the tract as well as possibly the behaviors of speech articulators (tongue, lips, jaw, and soft palate), yielding disorders of resonance and/or articulation (Bloomer, 1971; Darley, 1983). Yet the speech production system is remarkably flexible and adaptive and perceptually normal speech can be produced despite relatively large perturbations to the articulatory system (Bloomer, 1971; Darley, 1983; Subtelny and Mestre, 1964; Warren et al., 1980; Weinberg, 1968).

Many studies have explored speech production with the mandible fixed by a bite block. One of the earliest acoustic studies was that of Lindblom and Sundberg (1971) who developed an articulatory model for the production of the acoustic correlates of an inventory of vowels. They found that jaw position is highly deterministic of the first three vowel formants. As jaw opening increased, their model predicted an increase in formant frequencies, most notably in $F 1$. They tested these predictions by analyzing the spectra of Swedish vowels produced with unnaturally large interincisal separations (Lindblom et al., 1979). Spectra were analyzed from the first cycle of vocal fold vibration, to avoid the possibility that auditory feedback could be used for on-line compensation. Despite the large jaw openings, essentially normal vowel spectra were observed, presumably through compensatory tongue posturing. Because the compensation occurred before auditory feedback could be used for error correction, the data were interpreted to suggest that speech motor control processes use predictive control to develop new articulatory profiles appropriate for the changes in jaw position. Other early bite-block studies have replicated this finding of instantaneous compensation for the presence of a bite block (Gay et al., 1981; Kelso and Tuller, 1983; Lindblom et al., 1979; Lubker, 1979). In most of these investigations, however, differences between perturbed and unperturbed conditions were judged to be nonsignificant, but no statistical analyses were applied to the experimental data.

Subsequent observations have revealed that there may not be complete compensation for speech produced with a bite block (Flege et al., 1988; Fowler and Turvey, 1980). Researchers have reported small but measurable differences in formant frequencies of vowels produced with and without a bite block in place, and have reported that vowels produced under bite-block conditions suffer slightly in intelligibility (Flege et al., 1988; Fowler and Turvey, 1980).

More recently, attention has been focused on the production of consonants under conditions of perturbation. There is somewhat less flexibility in consonant production as compared to the production of vowels. Moreover, certain consonants appear to require greater articulatory precision than others. For example, Flege et al. (1988) have proposed that a complete constriction by the tongue may not be required for the production of a perceptually adequate [t], whereas a rather specific groove shape may be needed for accurate [s] production. Although these investigators reported deviations for both consonants and vowels in a bite block as compared to a jaw-free condition, more deviations from the norm were found for consonants in terms of acoustic, physiological, and 
perceptual measures (Flege et al., 1988). Further, compensation to the bite block in consonant production was not immediate, and in some subjects improvements in the accuracy of consonant production were observed after a $10-\mathrm{min}$ period of spontaneous speech with the bite block in place. These data were interpreted to suggest that sensory feedback provided for error-based correction. In other subjects the reverse was true: consonant production deviated even further from normal after the period of adaptation. Flege et al. (1988) concluded that these findings indicated overcompensating to the presence of the bite block. Although the Flege et al. (1988) study has suggested that complete compensation for fixation of the jaw does not occur for alveolar stop and fricative production, the effects of the bite block were not systematic, and there was considerable individual variability in compensatory articulation strategies to the presence of the bite block. Further research is warranted with a large subject pool before any definitive conclusions can be drawn.

Another interesting implication of the Flege et al. (1988) study concerns the role of sensory feedback in compensation. Clearly a fundamental question in speech motor control is to what extent, if any, somatosensory and/or auditory feedback interacts with central control signals in the production of speech (Smith, 1992). The results of perturbation experiments, such as those involving jaw fixation, may provide insights into the potential role of sensory feedback in the generation and modification of speech articulatory gestures. For example, immediate and complete compensation to increased jaw opening is consistent with the idea that predictive control processes operate without error-based correction (Borden, 1979; Kelso and Tuller, 1983). Alternatively, improvements in speech compensation to perturbation over time [as found in certain subjects of Flege et al. (1988)] supports a model incorporating sensory feedback in the speech adaptation process (Flege et al., 1988).

Adaptation to articulatory perturbation may be viewed as a developing system in which a new set of articulatory programs evolves for the change in oral function. Sensory feedback may be relatively more important in developing motor systems during these periods of skill acquisition (Smith, 1992). Auditory feedback appears crucial to normal speech sound acquisition and even relatively minor hearing loss in children may result in errors of speech and language (Osberger and McGarr, 1982). Sensory information that may be used to control speech production is also available from a variety of receptors located in oral-facial structures (Smith, 1992). The role of auditory and somatosensory inputs in the moment-to-moment corrections of speech gestures in the mature adult system is not clear. However, there is evidence from investigations of the speech of hearing impaired and aging individuals suggesting that certain parameters of speech and/or particular sound classes may require on-line monitoring (Slawinski, 1994; Waldstein, 1990). Further, it seems likely that particular sound classes, such as sibilants, may require greater articulatory precision (Flege et al., 1988; Gay et al., 1981; Stevens, 1972) and rely more heavily on feedback, particularly under conditions of articulatory perturbation.

In order to further examine the extent of compensation as well as the role of feedback in developing compensatory strategies in the present investigation, a variety of speech sound classes were tested under conditions of perturbation. Measurements were made of the vowels [i a u], the fricatives [s š], and the stop consonants [ $\mathrm{ptk}$ ] produced by 15 adult speakers of French in one free-mandible and two fixed mandible conditions (small and large bite block). Although direct comparison of adaptation for vowels versus consonants may not be feasible, it is interesting to explore general compensatory patterns across these phoneme classes. Speech acoustic data were recorded immediately upon bite-block insertion and after a 15-min accommodation period.

Based on the model and simulation data of Lindblom and Sundberg (1971), certain predictions can be made concerning the perturbing effects of increased jaw opening on vowel articulation. In the absence of compensation, an increase in jaw opening would yield higher vowel first formant frequencies due to decreased vocal tract constriction and increased front cavity cross-sectional area. $F 2$ values for [i] are expected to decrease somewhat, while little change is anticipated in $F 2$ of [a]. Target undershoot may be less likely with bite blocks that maintain normal jaw relationships but restrict movement (e.g., a small bite block in the production of the high vowels [i] and [u]). With respect to consonant production, an increase in jaw opening might be predicted to lower the spectral energy concentrations due to tongue retraction, incomplete vocal tract constriction, and increased front cavity dimensions (Flege et al., 1988). It might also be predicted that the duration of different acoustic segments may increase in response to perturbation (Hamlet, 1979; Tobey and Finger, 1983). Changes in the degree of compensation during the course of articulation or subsequent to a period of accommodation would provide support for models that incorporate sensory feedback in speech motor control (for a review, see Smith, 1992).

\section{METHOD}

\section{A. Subjects}

Fifteen adult female native speakers of (Québec) French, ranging in age from 20 to 33 years, participated in the experiment. All subjects were free from speech and/or language disorders and passed an audiometric screening $(<15 \mathrm{~dB} \mathrm{HL}$ at $0.5,1$, and $2 \mathrm{kHz}$ ). Speakers were also screened to ensure normal occlusal relationships.

\section{B. Stimuli}

The stimuli included the three vowels [i a u] produced in isolation, the voiceless stop consonants $[p t k]$ in the environment preceding the same three vowels, and the voiceless fricatives [s š] in the same vowel environments. Each stimulus was printed in orthographic form on a $3 \times 5$-in index card for presentation to the subjects. Ten repetitions of each stimulus were elicited in random order in a series of perturbed and unperturbed conditions.

\section{Procedure}

Two subtests were run on separate days over three different sessions: immediate compensation and postconversa- 
tion. The immediate compensation subtest included three conditions: jaw-free or normal (N), large bite block (LBB: $22.5 \mathrm{~mm}$ for vowels and $10 \mathrm{~mm}$ for CV stimuli) and small bite block (SBB: $2.5 \mathrm{~mm}$ for vowels and $5 \mathrm{~mm}$ for CV stimuli). For the postconversation subtest, only a single biteblock condition $(10 \mathrm{~mm}, \mathrm{BB})$ was compared to a jaw-free condition. The bite-block sizes were chosen based on previous research and simulations that showed that, without compensatory gestures, the spectral characteristics of the vowels and consonants produced under these bite-block conditions would be substantially altered (Lindblom and Sundberg, 1971; Linblom et al., 1979). Both large and small sizes were utilized for all productions in order to examine compensation under conditions that change normal jaw opening relationships (e.g., a large bite block for the high vowel [i] which normally requires a small opening) and those that restrict jaw movement but do not significantly alter typical jaw opening dimensions (e.g., a small bite block for [i]). For the postconversation subtest, we wanted to make as direct a comparison as possible to the immediate compensation conditions. However, it was not feasible to require subjects to speak for 15 min with a $22.5-\mathrm{mm}$ bite block in place. Therefore we selected a comfortable size jaw opening that was still predicted to affect the spectral characteristics of both vowels and consonants (Lindblom and Sundberg, 1971; Lindblom et al., 1979). Presentation of the blocks of vowel and CV stimuli were counterbalanced within each subtest. The stimuli in the postconversation condition were elicited following a 15-min period of conversation with the $10-\mathrm{mm}$ bite block in place to determine whether speakers would accommodate to the perturbation subsequent to a short period of practice. Bite blocks were fashioned out of dental material (Express putty) and adjusted for each speaker to ensure a vertical distance of $22.5,2.5,5$, or $10 \mathrm{~mm}$ measured at the incisors. Bite blocks were inserted and removed by the examiner for each trial (regardless of whether the subsequent trial required the same bite block).

Speakers' productions were recorded using a digital audio tape recorder (Sony DTC-57ES) and a directional microphone (Sennheiser MD421U) placed approximately 10-in in front of the speaker's mouth.

\section{Analyses}

Recordings were digitized at a rate of $10 \mathrm{k}$ samples/s for vowels and stops (with a $4.5-\mathrm{kHz}$ low-pass filter) and $20 \mathrm{k}$ for fricatives (with a 9-kHz low-pass filter) with 12-bit quantization using the BLISS speech analysis system (Mertus, 1989). Both temporal and spectral measures were computed for each stimulus. For each token, the duration of the target vowel or consonant was determined from the waveform display. Vowel durations were measured from the onset of voicing through the end of periodicity. Stop consonant onsets were demarcated by the burst associated with the stop release; the end of the consonant corresponded with the end of aspiration noise and the onset of periodicity associated with the following vowel. For fricatives, similar landmarks were utilized, with the onset corresponding to the onset of frication noise and the offset defined by the end of the noise segment and the onset of vocalic periodicity.
TABLE I. Acceptable frequency ranges for $F 1$ and $F 2$.

\begin{tabular}{ccr}
\hline \hline & $F 1$ & $F 2$ \\
\hline$[\mathrm{u}]$ & $200-500$ & $700-1600$ \\
{$[\mathrm{a}]$} & $600-900$ & $900-1600$ \\
{$[\mathrm{i}]$} & $200-500$ & $1700-2700$ \\
\hline \hline
\end{tabular}

In terms of spectral analyses, for vowel segments, the first two formant frequencies were measured at two distinct points in the waveform to examine the immediacy of compensation and possible "on-line" adjustments. A 25.6-ms full Hamming window was placed at the first glottal pulse of the vowel and the formants extracted via LPC analysis using a 14-pole network. To avoid spurious peaks, if the $F 1$ or $F 2$ values did not fall within an appropriate predetermined range (see Table I and Delattre, 1966; Peterson and Barney, 1952), the number of poles in the LPC algorithm was adjusted and the formants were recomputed. Values that remained out of range were excluded from the analyses (Baum and Katz, 1988). A second window was placed at the midpoint of the vowel and $F 1$ and $F 2$ values were comparably extracted. At this window placement, if the adjustment of LPC poles did not yield appropriate values, the window was shifted \pm 20 $\mathrm{ms}$ and the analysis recomputed. As above, frequency values outside the range limits were excluded (a total of $4 \%$ and $7 \%$ of the $F 1$ and $F 2$ values, respectively, across both window positions were eliminated via this procedure).

For stop and fricative consonants, the spectral measure utilized was the centroid frequency. The centroid represents a weighted average of the spectral peak frequencies and has been utilized to characterize, in part, the accuracy of consonant place of articulation (Baum and McNutt, 1990; Forrest et al., 1988; Nittrouer et al., 1989; Waldstein and Baum, 1991). Although the centroid frequency may not necessarily be associated with specific vocal tract cavity resonances, it does reflect the overall spectrum and can thus be used to assess the acoustic consequences of compensation or the lack thereof. Centroids were computed at the burst of the stop consonants and at the midpoint of the fricatives in order to capture the attributes of the consonants at a point least affected by vocalic environment.

\section{RESULTS}

\section{A. Immedlate compensation-Duration measures}

Mean durations for each of the vowel and consonant segments were computed in each condition; group mean values are displayed in Table II and significant differences between conditions are indicated. Three separate analyses of variance (ANOVAs) were conducted for the three segment types. Details of all results are provided in the Appendix; here only significant differences across conditions will be highlighted. As may be seen from the table, for [s], durations in the LBB condition were significantly shorter than those in the SBB and N conditions, which did not differ from one

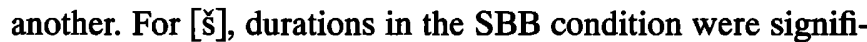
cantly longer than those in the $\mathrm{N}$ condition; durations in the LBB condition showed a nonsignificant trend in the same 
TABLE II. Mean durations in immediate compensation subtest for vowels, stops, and fricatives in three bite-block conditions. Values that differ significantly across bite-block (BB) conditions are indicated between columns.

\begin{tabular}{lccc}
\hline \hline & N & SBB & LBB \\
\hline Vowels & & & \\
[u] & 245 & 247 & 255 \\
[a] & 233 & 225 & 229 \\
[i] & 243 & 241 & 253 \\
Stops & & & \\
[p] & 40 & 41 & 41 \\
[t] & 50 & 51 & 51 \\
[k] & 66 & 69 & 68 \\
Fricatives & & & \\
[s] & 207 & 207 & 201 \\
[s] & 207 & 214 & 212 \\
\hline \hline
\end{tabular}

direction. Nevertheless, there was a great deal of individual variability in fricative duration values, with one-quarter to one-half of the subjects displaying patterns different from that of the group as a whole.

\section{B. Immediate compensation-Spectral measures}

For vowels, mean $F 1$ and $F 2$ values were calculated at both measurement points, with group mean values displayed in Table III. As shown, $F 1$ values tended to be higher in the LBB condition relative to the other two conditions at both windows. A measurement point (onset, midpoint) $\times$ vowel ([i a u]) $\times$ condition (N,SBB,LBB) ANOVA revealed that $F 1$ values at the onset were significantly higher than those measured at the vowel midpoint. Not unexpectedly, the $F 1$ values for [a] were significantly higher than those for [i] and [u]. As noted, post hoc tests revealed that $F 1$ frequencies in the LBB condition were significantly higher than those in the SBB and N conditions, which did not differ from one another. The magnitudes of the $F 1$ differences across LBB and $\mathbf{N}$ conditions exceeded the difference limens for those frequencies (Flanagan, 1955; Hawks, 1994; Kewley-Port and Watson, 1994).

For $F 2$ values, there is a similar pattern of significantly higher formant frequencies in the LBB condition relative to the other two conditions for [u] only. In contrast, for [i], F2 frequencies were significantly lower in the LBB condition as
TABLE IV. Mean stop and fricative consonant centroid frequencies in three bite-block conditions (immediate compensation subtest). Values that differ significantly across bite-block (BB) conditions are indicated between columns.

\begin{tabular}{|c|c|c|c|c|}
\hline & $\mathbf{N}$ & & SBB & LBB \\
\hline Stops & & & & \\
\hline [p] & 2674 & & 2634 & 2476 \\
\hline$[\mathrm{t}]$ & 3168 & & 3202 & 3114 \\
\hline [k] & 3040 & & 2937 & 2777 \\
\hline \multicolumn{5}{|c|}{ Fricatives } \\
\hline$[\mathrm{s}]$ & 6823 & $>$ & 6156 & 6196 \\
\hline$[\check{\mathbf{s}}]$ & 4845 & $>$ & 4426 & 4230 \\
\hline
\end{tabular}

compared to the $\mathrm{N}$ and SBB conditions. As with $F 1$ values, the LBB-N differences in $F 2$ exceeded perceptual difference limens (Flanagan, 1955; Hawks, 1994; Kewley-Port and Watson, 1994). There were no differences across the speaking conditions for [a].

Mean stop and fricative consonant centroid frequencies are presented in Table IV. A cursory examination of the table reveals lower centroid values in the LBB condition as compared to both $\mathbf{N}$ and SBB conditions for stop consonants. However, it should be noted that the centroid values for [t] are fairly comparable across LBB and N conditions; in fact, only seven of the individual subjects produced $[t]$ with lower centroids in the LBB as compared to the $\mathrm{N}$ condition. The fricative centroid frequencies appear to be higher in the $\mathrm{N}$ condition relative to both BB conditions.

\section{Postconversation-Duration measures}

Table $\mathrm{V}$ displays the mean duration values in the $\mathrm{BB}$ and $\mathrm{N}$ conditions for the vowel, stop, and fricative stimuli. The table reveals little, if any, influence of the BB on segment durations.

\section{Postconversation-Spectral measures}

Mean $F 1$ and $F 2$ frequencies computed at both measurement points are presented in Table VI, which shows little difference in average $F 1$ or $F 2$ values across conditions. It should be noted that the mean difference across conditions in

TABLE III. Mean $F 1$ and $F 2$ frequencies at both measurement points in three bite-block conditions (immediate compensation subtest). Values that differ significantly across bite-block (BB) conditions are indicated between columns.

\begin{tabular}{|c|c|c|c|c|c|c|c|c|}
\hline & \multicolumn{4}{|c|}{ Onset } & \multicolumn{4}{|c|}{ Midpoint } \\
\hline & $\mathbf{N}$ & SBB & & LBB & $\mathbf{N}$ & SBB & & LBB \\
\hline$F 1$ & & & $<^{a}$ & & & & $<^{a}$ & \\
\hline$[\mathrm{u}]$ & 337 & 334 & & 318 & 304 & 305 & & 307 \\
\hline [a] & 833 & 832 & & 925 & 787 & 800 & & 781 \\
\hline [i] & 229 & 302 & & 398 & 285 & 293 & & 322 \\
\hline \multicolumn{9}{|l|}{$F 2$} \\
\hline$[\mathrm{u}]$ & 837 & 813 & $<$ & 947 & 885 & 885 & & 842 \\
\hline [a] & 1371 & 1328 & & 1379 & 1294 & 1301 & & 1277 \\
\hline [i] & 2253 & 2240 & $>$ & 2270 & 2354 & 2385 & $>$ & 2192 \\
\hline
\end{tabular}

a Overall, $F 1$ values were higher in the LBB condition. No statistical comparisons for individual vowels were carried out because the vowel by condition interaction did not reach significance. 
TABLE V. Mean durations in postconversation subtest for vowels, stops, and fricatives in two bite-block conditions.

\begin{tabular}{ccc}
\hline \hline & N & BB \\
\hline Vowels & & \\
{$[\mathrm{u}]$} & 241 & 233 \\
{$[\mathrm{a}]$} & 216 & 221 \\
{$[\mathrm{i}]$} & 225 & 244 \\
Stops & & \\
{$[\mathrm{p}]$} & 39 & 42 \\
{$[\mathrm{t}]$} & 49 & 51 \\
{$[\mathrm{k}]$} & 66 & 67 \\
Fricatives & & \\
{$[\mathrm{s}]$} & 204 & 205 \\
{$[\mathbf{s}]$} & 203 & 208 \\
\hline \hline
\end{tabular}

F2 frequencies for [u] (only) surpassed the maximum estimated difference limen for that frequency range (Flanagan, 1955). No significant differences across conditions emerged in statistical analyses.

Mean stop and fricative consonant centroid frequencies are provided in Table VII. For all three stop consonants, centroid values in the BB condition are lower than in the $\mathrm{N}$ condition. However, only the differences for [p] and [k] reached statistical significance. As may be noted from the table, similar to stop consonants, fricative centroids were lower in the BB condition compared to the $\mathbf{N}$ condition. A fricative $\times$ condition ANOVA confirmed this pattern.

\section{E. Individual variability}

As a means of categorizing individual subjects' compensatory abilities as "good" or "imperfect," the spectral data for one vowel [i], one stop consonant [t], and both fricatives [s] and [š] were analyzed further as arbitrary exemplars of overall performance. ${ }^{1}$ For each subject, the differences in frequency $\left({ }^{\wedge} f\right)$ for consonant centroid values in the LBB and normal $(\mathrm{N})$ conditions were expressed as a percentage of the $\mathbf{N}$ centroid value [i.e., ${ }^{\wedge} \mathbf{f}=(\mathrm{LBB}-\mathrm{N}) / \mathrm{N} \times 100$ ] in both immediate compensation and postconversation conditions. A similar computation was derived for vowel $F 1$ and $F 2$ frequencies measured at vowel onset and vowel midpoint separately. Based in part on the maximum estimated difference limens for vowel formant frequencies (Flanagan, 1955), a cutoff of ${ }^{\wedge} \mathrm{f} \leqslant 5 \%$ was selected as the limit for the good compensation

TABLE VI. Mean $F 1$ and $F 2$ frequencies at both measurement points in two bite-block conditions (postconversation subtest).

\begin{tabular}{crrrrr}
\hline \hline & \multicolumn{3}{c}{ Onset } & & \multicolumn{2}{c}{ Midpoint } \\
\cline { 2 - 3 } \cline { 5 - 6 } & N & BB & & N & BB \\
\hline$F 1$ & & & & 318 & 306 \\
{$[\mathrm{u}]$} & 357 & 345 & & 812 & 805 \\
{$[\mathrm{a}]$} & 835 & 850 & & 295 & 296 \\
{$[\mathrm{i}]$} & 328 & 316 & & & \\
$F 2$ & & & & & \\
{$[\mathrm{u}]$} & 882 & 777 & & 882 & 816 \\
{$[\mathrm{a}]$} & 1281 & 1355 & & 1253 & 1281 \\
{$[\mathrm{i}]$} & 2347 & 2351 & & 2331 & 2396 \\
\hline \hline
\end{tabular}

TABLE VII. Mean stop, and fricative consonant centroid frequencies in two bite-block conditions (postconversation subtest). Values that differ significantly across bite-block (BB) conditions are indicated between columns.

\begin{tabular}{cccc}
\hline \hline & N & & BB \\
\hline Stops & & $>$ & \\
{$[\mathrm{p}]$} & 2684 & $>$ & 2508 \\
{$[\mathrm{t}]$} & 3185 & $>$ & 2748 \\
{$[\mathrm{k}]$} & 3085 & & \\
& & & 6052 \\
Fricatives & & & 4247 \\
{$[\mathrm{~s}]$} & 6626 & & \\
{$[\mathrm{~s}]$} & 4875 &
\end{tabular}

category. For vowels, both onset and midpoint ${ }^{\wedge} f$ values had to be $\leqslant 5 \%$ for the subject to qualify as a good compensator.

The results of these analyses revealed that in the immediate compensation condition, only two individual subjects were classified as exhibiting good compensation skills in the production of $F 1$ and only a single subject fell in the good category for $F 2$ (the same as one of the two above). In the postconversation condition, an additional subject improved for qualification in the good category for $F 1$ production; for $F 2$, three additional individuals were judged as good compensators, with two more just missing the 5\% cutoff. The remaining 9 of the 15 subjects were classified as exhibiting imperfect compensation even after the conversation accommodation period.

Results of the categorization procedure for $[t]$ yielded four individuals in the good category in both the immediate compensation and postconversation subtests, with an additional eight subjects classified as good in one of the two conditions. There was no evidence of consistent improvement after the accommodation period.

Finally, for the fricatives, three individuals were classified as good for [s] and two for [̌s] in both subtests. Subsequent to conversation, two subjects who had been categorized as imperfect in the immediate compensation subtest improved their performance to the good category for [s] production.

In general, the same individuals who exhibited good compensatory abilities for one sound category did so across the phonéne categories. Thus there appear to be individual differences in the ability to compensate for an articulatory perturbation such as mandibular fixation. An alternative explanation, however, is that the presence of a bite block may be less perturbing to subjects exhibiting good as opposed to imperfect compensation. Subject variables such as the size and morphology of the oral cavity, or differences in the degree to which a speaker uses the jaw to produce a target sound, may contribute to differences between subjects. Clearly, future research (as discussed below) is needed to clarify these and other issues related to individual differences in compensation.

\section{DISCUSSION}

A major finding of the present study was that speech compensation to the presence of a bite block was not complete. This was somewhat surprising given the results of 
most earlier studies of bite-block speech showing immediate compensation for increased jaw opening (Gay et al., 1981; Kelso and Tuller, 1983; Kelso et al., 1984; Lindblom and Sundberg, 1971; Lubker, 1979). A second important finding was that, at least for vowels, speech compensatory strategies may have developed over time, with normal acoustic parameters approached after a period of speech adaptation with the bite block in place. The effects of perturbation may be longer lasting for consonants, perhaps reflecting the greater articulatory precision necessary for accurate consonant as contrasted to vowel production.

\section{A. Immediate compensation}

The results of the current investigation revealed that the presence of a bite block did not significantly affect the durations of vowels or stop consonants. These data are consistent with the work of Flege et al. (1988) who reported no significant effects of a bite block on the duration of $\left[\mathrm{e}^{\mathrm{I}}\right]$ segments. In contrast, increased jaw opening did significantly affect fricative duration in the present study. The duration of [s] decreased in the LBB condition, while the duration of [气̌] increased in the SBB condition. These changes may reflect the perturbing effects of the bite-block and/or compensatory changes in articulation. However, "we hesitate to draw firm conclusions from these data because of the high degree of individual variability in the fricative duration measures.

Clearer evidence of the perturbing effects of increased jaw opening on speech articulation comes from the results of spectral analyses. The presence of the bite block significantly influenced vowel formant values; $F 1$ was significantly higher in the LBB as contrasted to the SBB and N conditions. These data are consistent with the work of Flege et al. (1988) who found significantly higher $F 1$ values of $\left[\mathrm{e}^{\mathrm{I}}\right]$ tokens produced under bite-block as opposed to jaw-free conditions. Similarly, Fowler and Turvey (1980; but cf. Gay et al., 1981; Kelso and Tuller, 1983; Kelso et al., 1984; Lindblom et al., 1979; Lindblom and Sundberg, 1971; Lubker, 1979) found near-significant differences in $F 1$ values of vowels produced under bite-block and normal conditions. It seems reasonable to assume that increased $F 1$ is due to decreased vocal tract constriction and increased front cavity cross-sectional area (Flege et al., 1988; Lindblom and Sundberg, 1971). It should be noted, however, that the differences found in the present investigation, like those reported by Fowler and Turvey (1980), were smaller than would have been expected had no compensation occurred. Further, although differences were found between first formant frequencies measured at vowel onset and vowel midpoint, the differences appeared in both normal and bite-block conditions. These data suggest that compensation, if any, for the presence of the bite block did not change during vowel production (Baum and Katz, 1988). That is, it seems unlikely that compensatory adjustments occurred "on-line" during vowel production. Evidence for error-based correction operating over a long time frame will be discussed below.

Our results also revealed that, only for the vowel $[\mathrm{u}], F 2$ values were significantly higher in the LBB condition as contrasted to both the N and SBB conditions. These data are consistent with the simulation and empirical data of Lind- blom and colleagues (Lindblom and Sundberg, 1971; Lindblom et al., 1979), showing the greatest change (increase) in $F 2$ with increased jaw opening for the vowel [u]. In contrast, $F 2$ frequencies were significantly lower in the LBB condition as compared to the N and SBB conditions for [i]. Although Lindblom and Sundberg (1971) did not predict a major change in $F 2$ across normal and bite-block conditions for [i], the pattern observed in their simulation data is similar to that observed in the present study - that is, a lower $F 2$ under conditions of perturbation. We found no significant difference in $F 2$ across speaking conditions for [a], and this is consistent with the simulation data of Lindblom and Sundberg (1971) showing relatively little change in $F 2$ of [a] with increased jaw opening.

It is interesting to note that overall the effects of perturbation were more evident in the LBB condition relative to the SBB condition. This finding is perhaps unexpected for the vowel [a], which is normally produced with a relatively large jaw opening. However, the $22.5-\mathrm{mm}$ large bite block is likely to have exceeded normal jaw opening magnitudes for most speakers; viewed in this light, the pattern of results for the two bite-block sizes is less surprising.

In summary, the results of the spectral analyses of vowels produced under conditions of perturbation revealed small but significant effects of the large bite block on formant values. These data suggest that compensation to the bite block was neither immediate nor complete, as measured by the acoustic parameters examined in this investigation (cf. Gay et al., 1981; Kelso and Tuller, 1983; Kelso et al., 1984; Lindblom and Sundberg, 1971; Lubker, 1979). The effects of increased jaw opening did not result in spectral differences as large as would be expected from the results of vocal tract simulation studies (Lindblom and Sundberg, 1971), suggesting that some adaptation or compensation to the bite block did occur. In fact, the SBB had no significant effects on vowel spectral characteristics, and individual subjects did exhibit good compensation. However, the average changes in vowel formants in the LBB condition exceeded perceptual difference limens for the relevant frequency ranges, indicating that they would be salient to listeners (Flanagan, 1955; Hawks, 1994; Kewley-Port and Watson, 1994). Recent investigations have shown that vowels produced under conditions of perturbation may suffer in intelligibility (Flege et al., 1988; Fowler and Turvey, 1980). Clearly, future investigations are needed to evaluate the quality and acceptability of the sounds produced under the various experimental conditions of the present study (Flege et al., 1988).

Further evidence of the perturbing effects of increased jaw opening on speech articulation can be seen in the results of spectral analysis of consonants. Consistent with the results of Flege et al. (1988), significantly lower centroid values were found for stop consonants produced in the LBB as contrasted to the SBB and N conditions. Incomplete vocal tract constriction and increased front cavity dimensions may have given rise to the lower centroid values in the bite-block condition of the present investigation. It is important to point out, however, that there was individual variability in compensatory skills evidenced in both studies. Flege et al. (1988) 
interpreted some of the intersubject variation as reflecting instances of overcompensation by certain individuals.

The results of the spectral analyses of fricatives in the present investigation also revealed lower centroid values in both LBB and SBB conditions when contrasted to N. The lower centroids may be due to tongue retraction and increased front cavity length (McGowan and Nittrouer, 1988), in accord with the significant differences in lingual articulatory configuration for [s] observed under conditions of perturbation by Flege et al. (1988).

\section{B. Postconversation}

As discussed above, the results of the analyses of the immediate compensation condition revealed that, overall, the compensation to increased jaw opening during speech was incomplete. Significant differences were observed in $F 1$ and $F 2$ values for vowels produced with the LBB in place. The results of the postconversation condition extend these findings and suggest that, for vowels, compensatory articulatory strategies may develop over time, and normal acoustic parameters may be approached after $15 \mathrm{~min}$ of conversation with a bite block in place. Our results revealed little if any influence of the presence of a bite block on vowel segment durations, and no significant differences were found in group mean vowel $F 1$ or $F 2$ between bite-block and normal conditions. $^{2}$ Nonetheless, there were individual subjects whose vowel productions did not change substantially following the conversation period.

It could be argued that the failure to find significant differences between the bite-block and normal trials could be due to the fact that the $10-\mathrm{mm}$ bite block used in this condition may not have been sufficiently perturbing to vowel articulation. It is true that in the immediate compensation condition, the differences in vowel formant values between biteblock and normal conditions were small. A smaller bite block may give rise to even smaller differences, and this could explain the failure to find significant differences between bite-block and normal conditions for vowel formant values. However, based on the simulation data provided by Lindblom and Sundberg (1971), we can assume that the presence of a 10-mm bite block would give rise to significant changes in $F 1$ and $F 2$ in the absence of compensation. The small and nonsignificant differences found in the postconversation data, therefore, could indicate that compensatory articulation strategies developed over time during the period of conversation. Flege et al. (1988) also found that for $\left[\mathrm{e}^{\mathrm{I}}\right]$, normal acoustic parameters were approached after $10 \mathrm{~min}$ of conversation with the bite block in place; they hypothesized that sensory feedback may have provided for error-based correction of articulatory gestures (Flege et al., 1988). Our data support this interpretation and the potential role of sensory feedback in the development of speech compensatory processes.

In contrast to vowel production, spectral analyses of stops and fricatives revealed significant differences between bite-block and normal conditions even after the period of conversation with the bite block in place. For the three stop consonants and two fricatives, the centroid values were significantly lower in the bite-block as contrasted to the jaw- free condition. Again, certain subjects showed good compensation during the production of these consonants. The group data suggested that, although the accuracy of consonant production may have been improving during the period of conversation, complete compensation for increased jaw opening did not occur. Flege et al. (1988) also observed, in some subjects, persistent deviations from normal in the acoustic parameters of consonants after a $10-\mathrm{min}$ period of speech adaptation with the bite block in place.

The results of the postconversation condition reveal striking differences between the vowel and consonant data. In contrast to vowels, normal consonantal acoustic parameters were not observed after a 15 -min period of speech adaptation. We interpret these data as suggesting that speech sounds of different phoneme classes may be differentially affected by the presence of the bite block, because some may require greater articulatory precision for accurate production. Perhaps there is less flexibility in consonant as compared to vowel articulation. It must be pointed out, however, that the postconversation conditions for the vowels and consonants are not directly comparable and conclusions must be drawn cautiously. Recall that a $10-\mathrm{mm}$ bite block was used for both sound classes; this represents an identical size to that used for the consonants in the immediate compensation subtest. However, it is a smaller bite block than the LBB used for the vowels in the immediate compensation subtest. Yet, as noted earlier, the $10-\mathrm{mm}$ bite block would have influenced the spectral characteristics of vowels in the absence of compensation.

It seems reasonable to assume, therefore, that consonants may require a relatively longer period of speech adaptation than do vowels. In this regard it is interesting to note that artificial palates placed in the mouths of otherwise normal speakers require a lengthy adaptation period, perhaps involving days or weeks before normal consonant production is approached (Hamlet and Stone, 1974). Although it is impossible to leave bite blocks in place for long periods of time, further studies could be designed to look more closely at the adaptation period to ascertain the time required for various aspects of compensation to occur.

In general, the present results support a role for sensory feedback in speech production (Smith, 1992). Theories of speech motor control that rely solely on predictive control (e.g., Lindblom et al., 1979) cannot easily account for improvements over time in compensation for vowels produced with a bite block. Moreover, variability in the extent of compensation across individuals suggests that speakers may differ in their abilities to use various sensory feedback channels for speech adaptation (Flege et al., 1988).

Although a great deal of information may be gained from acoustic analyses, we would be aided in our assessment of the mechanisms involved in the speech adaptation process by the simultaneous recording of both acoustic and physiological parameters of vowels and consonants produced under conditions of perturbation (Flege et al., 1988; Kelso et al., 1984). Recording patterns of movement and muscle activity of the speech articulators may also provide insights into individual differences in speech compensatory strategies to articulatory perturbation. Variations in vocal tract dimen- 
sions and configurations-measurable only through articulatory-kinematic means-would surely affect compensatory abilities. Discovering subject variables that influence compensatory abilities has obvious clinical implications, as there are a variety of conditions that require speech adaptation to changes in oral form. Further, our data and those of Flege et al. (1988) suggest that speech adaptation may be learned over time, and compensation may be subject to therapeutic intervention.

\section{CONCLUSIONS}

In conclusion, the present data suggest that compensation to increased jaw opening during speech is neither as immediate nor as complete as previously hypothesized. Further, our data suggest that, at least for vowels, speech compensatory strategies may develop over time, perhaps involv- ing error-based correction. Consonants appear to require a more lengthy period of speech adaptation, and this may be due to the articulatory requirements for their accurate production. The present findings are consistent with theories of speech motor control that incorporate a role for sensory feedback and that allow for individual differences in compensatory abilities.

\section{ACKNOWLEDGMENTS}

We would like to thank M. Bourque, G. Devine, $M$. Gagner, S. Gagnon, and D. Israel for their assistance in data collection and analysis. We also thank the editor and reviewers for their comments. This work was supported in part by grants from the Fonds de la recherche en santé du Québec and the Medical Research Council of Canada.

\section{APPENDIX: SIGNIFICANT ANOVA RESULTS}

Immediate compensation $\quad \fallingdotseq$ Main effects

\section{Duration}

$\mathrm{V} \times$ condition

$\mathrm{S} \times$ condition

F $\times$ condition

Vowel F1

Meas. $\times \mathrm{V} \times$ condition

\section{Vowel F2}

Meas. $\times \mathrm{V} \times$ condition

\section{Stop centroid}

$\mathrm{S} \times$ condition

Fricative centroid

$\mathrm{F} \times$ condition

Postconversation

\section{Duration}

$\mathrm{V} \times$ condition

$\mathrm{S} \times$ condition

$\mathbf{F} \times$ condition

\section{Vowel F1}

Meas. $\times \mathrm{V} \times$ condition

\section{Vowel F2}

Meas. $\times \mathrm{V} \times$ condition

\section{Stop centroid}

$\mathrm{S} \times$ condition

Fricative centroid

$\mathrm{F} \times$ condition

$$
\begin{aligned}
& \text { V: } F(2,26)=5.227, p<0.02 \\
& \text { S: } F(2,26)=63.028, p<0.001 \\
& \text { F: } F(1,13)=6.364, p<0.05
\end{aligned}
$$$$
\text { F } \times \text { cond.: } F(2,26)=4.319, p<0.05
$$

meas.: $F(1,8)=7.364, p<0.05^{\mathrm{a}}$

$\mathrm{V}: F(2,16)=359.243, p<0.001$ cond.: $F(2,16)=10.685, p<0.001$

V: $F(2,10)=169.627, p<0.001$ V×cond.: $F(4,20)=6.184, p<0.002$

S: $F(2,26)=28.671, p<0.001$ cond.: $F(2,26)=15.027, p<0.001$

F: $F(1,13)=271.083, p<0.001$ cond.: $F(2,26)=23.843, p<0.001$
$\mathrm{V} \times$ cond.: $F(2,26)=4.910, p<0.02$
$\mathrm{~S} \times$ cond.: $F(2,28)=51.814, p<0.001$

V Xcond.: $F(2,22)=802.157, p<0.001$

$\mathrm{V} \times$ cond.: $F(2,12)=524.781, p<0.001$

S $\times$ cond.: $F(2,28)=5.062, p<0.02$
$\mathrm{S}: F(2,28)=23.422, p<0.001$ F: $F(1,14)=451.243, p<0.001$
cond.: $F(1,14)=22.214, p<0.001$ F: $F(1,14)=451.243, p<0.001$
cond.: $F(1,14)=22.214, p<0.001$ cond.: $F(1,14)=21.861, p<0.001$

$$
\mathrm{V}: F(2,22)=580.140, p<0.001
$$

V: $F(2,12)=95.422, p<0.001$ cond.: $F(1,6)=143.214, p<0.001$

\footnotetext{
${ }^{a}$ Each of the ANOVAs may have different degrees of freedom due to missing values in various cells. Thus the ANOVA results are based on (sometimes) as few as 6 of the 15 subjects tested. Legend: $V=$ vowel, $S=$ stop, $F=$ fricative, meas. = measurement point, cond.= condition.
} 
${ }^{1}$ Because of the large amount of intrasubject variability, we decided to choose a representative speech sound for the vowel and stop classes. The vowel [i] and stop [t] were selected in part because they have been included in previous investigations of compensatory articulation and thus provide a point of comparison with other acoustic data.

${ }^{2}$ It is important to recognize the fact that such an interpretation is based on the failure to find statistically significant differences between the bite-block and normal speech conditions. Conclusions must therefore be drawn cautiously.

Abbs, J. (1973). "Some mechanical properties of lower lip movement during speech production," Phonetica 28, 65-75.

Abbs, J., and Netsell, R. (1973). "An interpretation of jaw acceleration during speech as a muscle forcing function," J. Speech Hear. Res. 16, $421-425$.

Abbs, J. H. (1986). "Invariance and variability in speech production: a distinction between linguistic intent and its neuromotor implementation," in Invariance and Variability in Speech Processes, edited by J. S. Perkell and D. H. Klatt (Erlbaum, Hillsdale, NJ), pp. 202-225.

Baum, S. R., and Katz, W. F. (1988). "Acoustic analysis of compensatory articulation in children," J. Acoust. Soc. Am. 84, 1662-1668.

Baum, S. R., and McNutt, J. C. (1990). "An acoustic analysis of frontal misarticulation of /s/ in children," J. Phon. 18, 51-63.

Bloomer, H. H. (1971). "Speech defects associated with dental malocclusions and related abnormalities," in Handbook of Speech Pathology and Audiology, edited by L. E. Travis (Appleton Century Crofts, New York).

Borden, G. (1979). "An interpretation of feedback in established and developing speech," in Speech and Language: Advances in Basic Research, edited by N. Lass (Academic, New York), Vol. 3, pp. 223-242.

Darley, F. (1983). "Speech pathology," in Diagnosis and Treatment in Prosthodontics, edited by W. R. Laney and J. A. Gibilisco (Lea and Febiger, Philadelphia), pp. 346-376.

Delattre, P. (1966). Studies in French and Comparative Phonetics (La Haye).

Flanagan, J. (1955). "A difference limen for vowel formant frequency," J. Acoust. Soc. Am. 27, 613-617.

Flege, J. E., Fletcher, S. G., and Homiedan, A. (1988). "Compensating for a bite block in /s/ and / $/$ / production: Palatographic, acoustic, and perceptual data," J. Acoust. Soc. Am. 83, 212-228.

Forrest, K., Weismer, G., Milenkovic, P., and Dougall, R. (1988). "Statistical analysis of word-initial voiceless obstruents: Preliminary data," J. Acoust. Soc. Am. 28, 303-310.

Fowler, C., and Turvey, M. (1980). "Immediate compensation in bite-block speech," Phonetica 37, 306-326.

Gay, T., Lindblom, B., and Lubker, J. (1981). "Production of bite-block vowels: Acoustic equivalence by selective compensation," J. Acoust. Soc. Am. 69, 802-810.

Hamlet, S., and Stone, M. (1974). "Reorganization of speech motor patterns following prosthodontic changes in oral morphology," Speech Communication Seminar 2, 79-86.

Hamlet, S., and Stone, M. (1976). "Compensatory vowel characteristics resulting from the presence of different types of experimental dental prostheses," J. Phon. 3, 199-218.

Hamlet, S. L. (1979). "Physiological control of sibilant duration: Insights afforded by speech compensation to dental prostheses," J. Acoust. Soc. Am. 65, 1276-1285.

Hawks, J. W. (1994). "Difference limens for formant patterns of vowel sounds," J. Acoust. Soc. Am. 95, 1074-1084.

Hughes, O., and Abbs, J. (1976). "Labial-mandibular coordination in the production of speech: Implications for the operation of motor equivalence," Phonetica 33, 199-221.
Kelso, J. A. S., Tuller, B., Vatikiotis-Bateson, E., and Fowler, C. A. (1984). "Functionally specific articulatory adaptation to jaw perturbations during speech: Evidence for coordinative structures," J. Exp. Psychol. 10, 812832.

Kelso, J. A. S., and Tuller, B. (1983). "Compensatory articulation under conditions of reduced afferent information: A dynamic formulation," $J$. Speech Hear. Res. 26, 217-224.

Kewley-Port, D., and Watson, C. S. (1994). "Formant-frequency discrimination for isolated English vowels," J. Acoust. Soc. Am. 95, 485-496.

Lindblom, B., and Sundberg, J. (1971). "Acoustical consequences of lip, tongue, jaw, and larynx movement," J. Acoust. Soc. Am. 50, 1166-1179.

Lindblom, B., Lubker, J. F., and Gay, T. (1979). "Formant frequencies of some fixed-mandible vowels and a model of speech motor programming by predictive simulation," J. Phon. 7, 147-161.

Lubker, J. (1979). "The reorganization times of bite-block vowels," Phonetica 36, 273-293.

MacNeilage, P. (1970). “Motor control of serial ordering of speech," Psychol. Rev. 77, 182-196.

McGowan, R. S., and Nittrouer, S. (1988). "Differences in fricative production between children and adults: Evidence from an acoustic analysis of $/ \mathbb{S} /$ and /s/," J. Acoust. Soc. Am. 83, 229-236.

Mertus, J. (1989). BLISS User's Manual (Providence).

Nittrouer, S., Studdert-Kennedy, M., and McGowan, R. S. (1989). "The emergence of phonetic segments: Evidence from the spectral structure of fricative-vowel syllables spoken by children and adults," J. Speech Hear. Res. 32, 120-132.

Osberger, M. J., and McGarr, N. S. (1982). "Speech production characteristics of the hearing impaired," in Speech and Language: Advances in Basic Research and Practice, edited by N. J. Lass (Academic, New York), pp. 221-283.

Peterson, G. E., and Barney, H. L. (1952). "Control methods used in a study of the vowels," J. Acoust. Soc. Am. 24, 175-184.

Slawinski, E. B. (1994). "Acoustic correlates [b] and [w] produced by normal young to elderly adults," J. Acoust. Soc. Am. 95, 2221-2230.

Smith, A. (1992). "The control of orofacial movements in speech," Crit. Rev. Oral Biol. Med. 3. 233-267.

Stevens, K. N. (1972). "The quantal nature of speech: evidence from articulatory-acoustic data," in Human Communication: A unified view, edited by E. E. David and P. B. Denes (McGraw-Hill, New York), pp. 5166.

Subtelny, J. D., and Mestre, J. C. (1964). "Comparative study of normal and defective articulation of $/ \mathrm{s} /$ as related to malocclusion and deglutition," $\mathrm{J}$. Speech Hear. Disord. 29, 269-285.

Tobey, E. A., and Finger, I. M. (1983). "Active versus passive adaptation: an acoustic study of vowels produced with and without dentures," J. Prosthet. Dent. 49, 314-320.

Waldstein, R., and Baum, S. R. (1991). "Anticipatory coarticulation in the speech of profoundly hearing-impaired and normally hearing children," $\mathrm{J}$. Speech Hear. Res. 34, 1276-1285.

Waldstein, R. S. (1990). "Effects of postlingual deafness on speech production: Implications for the role of auditory feedback," J. Acoust. Soc. Am. 88, 2099-2114.

Warren, D. W., Nelson, G. R., and Allen, G. (1980). "Effects of increased vertical dimension on size of constriction port and fricative sound intelligibility," J. Acoust. Soc. Am. 67, 1828-1831.

Warren, D. W., Allen, G., and King, H. A. (1984). "Physiologic and perceptual effects of induced anterior open bite," Folia Phoniatr. 36, 164-173.

Weinberg, B. (1968). "A cephalometric study of normal and defective /s/ articulation and variations in incisor dentition," J. Speech Hear. Res. 11, 288-300. 